\title{
Upward trend in follicular lymphoma among the Korean population: 10-year experience at a large tertiary institution
}

\author{
Meejeong $\mathrm{Kim}^{1}$, Hee Sang Hwang ${ }^{1}$, Hyungwoo Cho ${ }^{2}$, Dok Hyun Yoon ${ }^{2}$, \\ Cheolwon Suh ${ }^{2}$, Chan Sik Park ${ }^{1}$, Heounjeong Go ${ }^{1}$, Jooryung Huh ${ }^{1}$ \\ Departments of ${ }^{1}$ Pathology and ${ }^{2}$ Oncology, Asan Medical Center, University of Ulsan College of Medicine, Seoul, Korea
}

\begin{abstract}
Background: Follicular lymphoma (FL) is the second most common non-Hodgkin lymphoma (NHL) in Western countries. However, it is relatively rare in Asia. This study examined epidemiologic characteristics of FL in South Korea, with an emphasis on recent trends of increase in cases. Methods: We retrospectively examined 239 cases of newly diagnosed FL at a large tertiary institution in Korea (Asan Medical Center, Seoul, Republic of Korea) between 2008 and 2017. Age-adjusted incidence rates and clinicopathological variables were analyzed, and joinpoint regression analysis was used to identify the changes. Results: The age-adjusted incidence of FL significantly increased during the study period $(p=.034)$, and the ratio of (relative incidence) patients with FL to patients with $\mathrm{NHL}$ increased from $4.28 \%$ to $9.35 \%$ in the same period. Over the 10 -year study assessment duration, the proportion of patients with stage III/IV FL $(p=$ .035) and expression of BCL2 ( $p=.022)$ or BCL6 $(p=.039)$ significantly increased. From 2013-2017, the proportion of patients with highrisk Follicular Lymphoma International Prognostic Index (FLIPI) score increased (21.5\% to $28.7 \%$ ), whereas that of low-risk FLIPI decreased $(55.4 \%$ to $38.6 \%)$, although those results were not statistically significant $(p=.066)$. Conclusions: We found an increasing incidence of FL, with a disproportionate increase in the incidence of high-stage disease and recent changes in the clinicopathologic features of the Korean patient population.
\end{abstract}

Key Words: Follicular lymphoma; Korea; Follicular Lymphoma International Prognostic Index (FLIPI); BCL2; BCL6

Received: April 13, 2021 Revised: June 26, 2021 Accepted: July 25, 2021

Corresponding Author: Jooryung Huh, MD, PhD, Department of Pathology, Asan Medical Center, University of Ulsan College of Medicine, 88 Olympic-ro 43-gil, Songpa-gu, Seoul 05505, Korea

Tel: +82-2-3010-4553, Fax: +82-2-472-7898, E-mail: jrhuh@amc.seoul.kr

Corresponding Author: Heounjeong Go, MD, PhD, Department of Pathology, Asan Medical Center, University of Ulsan College of Medicine, 88 Olympic-ro 43-gil, Songpa-gu, Seoul 05505, Korea

Tel: +82-2-3010-5888, Fax: +82-2-472-7898, E-mail: damul37@amc.seoul.kr

Follicular lymphoma (FL) is the second most common nonHodgkin lymphoma (NHL) in Western countries and accounts for $20 \%$ to $35 \%$ of all lymphomas [1,2]. In Asia, until recently, the incidence has been characteristically low, at approximately $5 \%$ to $10 \%$ of all lymphomas in most studies [3,4]. However, the relative proportion of $\mathrm{FL}$ in many Asian populations has increased rapidly in recent years, and FL is now the second most common low-grade B-cell lymphoma after mucosa-associated lymphoid tissue lymphoma in Asia [3,5-7]. The proportion of FL to NHL increased from $8.1 \%$ to $23.5 \%$ in China and $5 \%$ to $20 \%$ in Japan $[3,4,7,8]$; additionally, an increase in the proportion in Korea, from 3.4\% to 4.8\% between 1999 and 2012, has been reported $[9,10]$.

FL is characterized by indolent clinical behavior, and most cases involve a low histologic grade and low-risk Follicular Lymphoma International Prognostic Index (FLIPI), which is determined based on the sum of points assigned for each of the following risk factors: age $>60$ years, elevated lactate dehydrogenase (LDH) level, hemoglobin (Hb) level $<12 \mathrm{~g} / \mathrm{dL}$, Ann Arbor stage III/IV, and involvement of more than four nodal sites. Grade 3B FL or transformation to high-grade B-cell lymphoma significantly reduces survival $[11,12]$. However, due to the availability and broad use of rituximab, the incidence of transformation has been reduced, and the distribution of clinicopathologic features including histologic grade and FLIPI subgroup has changed, although the differences have not been reported consistently [12-14]. Most of the information in the literature is based on studies involving Caucasian patients, and characteristics of FL in East Asians have 
not been delineated well. In light of the recent increase in the incidence of FL in the Korean population, we investigated the trends in incidence of FL from 2008 to 2017, using data from a large tertiary institution in South Korea, and examined the clinicopathologic features of FL in the Korean population.

\section{MATERIALS AND METHODS}

\section{Age-adjusted incidence rate of $F L$ and frequency of $F L$ and} $\mathrm{NHL}$

The age-adjusted incidence rate, which is a weighted average of the age-specific crude rate, was calculated using data from Korean Statistical Information Service (http://kosis.kr/index/index.do). The age-adjusted incidence rates per 100,000 were calculated by dividing the annual number of FL cases observed in a specific age group of patients treated at Asan Medical Center between 2008 and 2017, by the number of corresponding person-years of observation; the obtained result was multiplied by 100,000.

Additionally, we retrieved the total numbers of inpatients and of patients with NHL or FL treated at Asan Medical Center, Seoul, between 2008 and 2017, using the Asan BiomedicaL research Environment, i.e., ABLE, research information retrieval system. The annual frequency of patients with NHL or FL was calculated using the date of initial diagnosis.

\section{Patient selection}

A total of 239 patients diagnosed with FL between 2008 and 2017, at Asan Medical Center was included in this retrospective study. The study included patients from whom excisional ( $\mathrm{n}=$ $215)$ and needle $(n=24)$ biopsy specimens were collected before treatment. The following demographic and clinical data were collected by reviewing the patients' medical records: age, sex, presence or absence of B symptoms (fever, night sweats, and weight loss), transformation to diffuse large B-cell lymphoma (DLBCL) (during follow-up), complete blood count, serum LDH level, bone marrow involvement, Ann Arbor stage, FLIPI risk group, and treatment modalities.

\section{Pathological review and immunohistochemistry data}

All cases were reviewed, and based on the revised 4th edition of the World Health Organization classification criteria, patients with testicular FL, duodenal-type FL, and pediatric-type FL were excluded [2]. The following pathological parameters were recorded: histologic grade (grades 1-2, grade 3A, and grade 3B), histologic pattern (follicular, follicular and diffuse, and diffuse), and immunophenotype (expression of BCL2, BCL6, CD10, or
Ki-67). Immunohistochemical (IHC) staining was performed on whole slides using a fully automated IHC assay on a Ventana BenchMark XT Autostainer (Ventana Medical Systems, Woonsocket, RI, USA). Antibodies specific for the following markers were used: CD3 (clone PS1, mouse mAb, Novocastra, Newcastleupon-Tyne, UK), CD5 (clone 4C7, mouse mAb, Novocastra), CD20 (clone L26, mouse mAb, Novocastra), Ki-67 (mouse mAb, Dako, Glostrup, Denmark), CD10 (clone 56C6, mouse mAb, Novocastra), CD21 (clone 2G9, mouse mAb, Novocastra), BCL2 (clone E17, rabbit mAb, Cell Marque, Rocklin, CA, USA), and BCL6 (clone GI191E/A8, mouse mAb, Cell Marque). Immunostained slides from $239 \mathrm{FL}$ cases were reviewed, and immunopositivity was defined as protein expression by $\geq 30 \%$ of the tumor cells [15-18]. Ki-67 proliferation index (PI) was assessed manually in neoplastic follicles, and a Ki-67 PI $\geq 30 \%$ was deemed as high expression [19-23].

\section{Statistical analyses}

Joinpoint regression analysis was performed to identify the changes in the incidence rates of FL at Asan Medical Center, using the Joinpoint Trend Analysis Software (ver. 4.8.0.1, Statistical Methodology and Application Branch, Surveillance Research Program, Division of Cancer Control and Population Sciences, National Cancer Institute). Joinpoint regression analysis usually is applied to study varying trends over time to identify the time point(s) at which the trends show significant changes [24-26]. Based on the trend analysis, we estimated the annual percentage change (APC) in the rates between trend-change points and the average annual percentage change (AAPC) for the entire study period. When there was no joinpoint (no significant change in trend), the APC was equal to AAPC.

Simple linear regression analysis was conducted to investigate changes in the proportion of clinicopathologic features of FL over the study period. Chi-square and Fisher exact tests were performed to evaluate the changes in clinicopathologic characteristics from 2008 to 2012, and from 2013 to 2017. In addition, overall survival (OS) was analyzed using the Kaplan-Meier method from the date of diagnosis to the date of death or the last followup visit, and data were compared using the log-rank test. All statistical analyses were conducted using SPSS software package ver. 18.0.0 (SPSS Statistics software, SPSS Inc., Chicago, IL, USA) and R ver. 3.6.3 (R Foundation for Statistical Computing, Vienna, Austria). Statistical significance was defined by $\mathrm{p}$-value $<.05$. 


\section{RESULTS}

\section{Incidence of FL between 2008 and 2017}

In total, 8,406 patients ( $0.17 \%$ of total inpatients) with NHL were treated at Asan Medical Center between 2008 and 2017, and 498 patients $(0.01 \%$ of total inpatients and $5.92 \%$ of $\mathrm{NHL})$ had FL. The proportion of FL patients (patients with FL/total inpatients) increased from $0.01 \%$ in 2008 to $0.02 \%$ in 2017, and the ratio of patients with FL to patients with NHL increased from $4.28 \%$ (26/607 cases) in 2008 to $9.35 \%$ (103/1102 cases) in 2017 (Supplementary Fig. S1).

Fig. 1 presents the increasing trend in the age-adjusted incidence rates of FL at Asan Medical Center between 2008 and 2017, and one joinpoint that was identified in 2010; the fastest increasing trend was observed in 2008-2010 (APC, 48.3; 95\% confidence interval [CI], 9.0 to 101.7) and a moderately increasing trend was observed in 2010-2017 (APC, 10.1; 95\% CI, 5.7 to 14.8) $(\mathrm{p}=.034)$ (Fig. 1).

\section{Clinicopathologic characteristics during defined periods}

The patients with FL ( $\mathrm{n}=239)$ included in this study had a median age of 52.1 years (range, 17 to 82 years) with a male:female ratio of 1.13:1. The overall clinicopathologic characteristics and initial treatment modalities used for the 239 patients and changes in the baseline characteristics from 2008 to 2012 and 2013 to 2017 are summarized in Table 1. The proportion of patients aged $>60$ years significantly increased from $13.2 \%$ in 2008 2012 to 34.5\% in 2013-2017 ( $\mathrm{p}=.001$ ) (Table 1). In 2013-2017, the proportion of patients with high-risk FLIPI (21.5\% to $28.7 \%)$

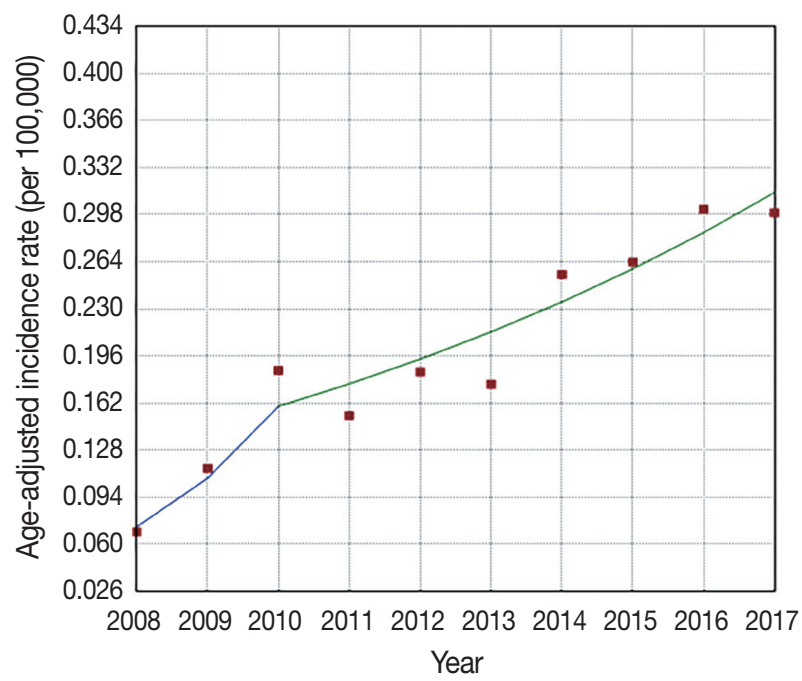

Fig. 1. Age-adjusted incidence rates of follicular lymphoma between 2008 and 2017 and grade 3B FL (5.9\% to 14.0\%) increased but not at a statistically significant level $(\mathrm{p}=.270$ and $\mathrm{p}=.077$, respectively) (Table 1 ).

Fig. 2 outlines the changes in the proportion of patients $>60$ years of age, with $\mathrm{Hb}$ level $<12 \mathrm{~g} / \mathrm{dL}$, elevated LDH level, and involvement of more than four nodal sites, which were the factors included in FLIPI score. The proportion of patients aged > 60

Table 1. Baseline patient characteristics

\begin{tabular}{|c|c|c|c|c|}
\hline \multirow[b]{2}{*}{ Characteristic } & \multirow[b]{2}{*}{$\begin{array}{c}\text { Total } \\
(n=239)\end{array}$} & \multicolumn{2}{|c|}{ Year } & \multirow[b]{2}{*}{$\begin{array}{c}\mathrm{p}- \\
\text { value }\end{array}$} \\
\hline & & $\begin{array}{c}2008-2012 \\
(n=68)\end{array}$ & $\begin{array}{c}2013-2017 \\
(n=171)\end{array}$ & \\
\hline Age $>60 y r$ & $68(28.6)$ & $9(13.2)$ & $59(34.5)$ & .001 \\
\hline Sex & & & & .267 \\
\hline Male & $127(53.1)$ & $40(58.8)$ & $87(50.9)$ & \\
\hline Female & $112(46.9)$ & $28(41.2)$ & $84(49.1)$ & \\
\hline B symptom & & & & .751 \\
\hline Absent & 212 (90.2) & $62(91.2)$ & $150(89.8)$ & \\
\hline Present & $23(9.8)$ & $6(8.8)$ & 17 (10.2) & \\
\hline $\mathrm{Hb}<12 \mathrm{~g} / \mathrm{dL}$ & $41(17.2)$ & $12(17.9)$ & $29(17.0)$ & .861 \\
\hline Elevated LDH & $46(19.8)$ & $15(23.8)$ & $31(18.3)$ & .353 \\
\hline Ann Arbor stage & & & & .111 \\
\hline Stage $1 / \|$ & $90(37.7)$ & $31(45.6)$ & $59(34.5)$ & \\
\hline Stage III/IV & $149(62.3)$ & $37(54.4)$ & $112(65.5)$ & \\
\hline Bone marrow involvement & $87(37.8)$ & $24(35.8)$ & $63(38.7)$ & .688 \\
\hline FLIPI & & & & .270 \\
\hline Low to intermediate & $173(73.3)$ & $51(78.5)$ & $122(71.3)$ & \\
\hline High & $63(26.7)$ & $14(21.5)$ & $49(28.7)$ & \\
\hline Histologic grade & & & & .077 \\
\hline $1-2 / 3 A$ & 211 (88.3) & $64(94.1)$ & $147(86.0)$ & \\
\hline $3 \mathrm{~B}$ & $28(11.7)$ & $4(5.9)$ & $24(14.0)$ & \\
\hline Histologic pattern & & & & .018 \\
\hline Follicular & $207(87.7)$ & $56(82.4)$ & $151(89.9)$ & \\
\hline Follicular and diffuse & $20(8.5)$ & $11(16.2)$ & $9(5.4)$ & \\
\hline Diffuse & $9(3.8)$ & $1(1.5)$ & $8(4.8)$ & \\
\hline BCL2 expression & & & & .086 \\
\hline Negative & $25(11.3)$ & $11(16.9)$ & $14(8.9)$ & \\
\hline Positive & $197(88.7)$ & $54(83.1)$ & $143(91.1)$ & \\
\hline BCL6 expression & & & & .002 \\
\hline Negative & $10(4.3)$ & $7(11.1)$ & $3(1.8)$ & \\
\hline Positive & $222(95.7)$ & $56(88.9)$ & $166(98.2)$ & \\
\hline CD10 expression & & & & .461 \\
\hline Negative & 44 (18.8) & $14(21.9)$ & $30(17.6)$ & \\
\hline Positive & $190(81.2)$ & $50(78.1)$ & $140(82.4)$ & \\
\hline $\mathrm{Ki}-67 \mathrm{PI} \geq 30 \%$ & $86(37.9)$ & $15(25.0)$ & $71(42.5)$ & .016 \\
\hline Treatment & & & & .049 \\
\hline Watch-and-wait & $44(18.4)$ & $6(8.8)$ & $38(22.2)$ & \\
\hline Rituximab-based & $130(54.4)$ & $40(58.8)$ & $90(52.6)$ & \\
\hline Radiation monotherapy & $30(12.9)$ & $11(16.4)$ & $19(11.5)$ & \\
\hline Transformation to DLBCL & $19(8.0)$ & $5(7.4)$ & $14(8.2)$ & .821 \\
\hline Deaths & $15(6.6)$ & $6(9.0)$ & $9(5.7)$ & .387 \\
\hline
\end{tabular}

Values are presented as number (\%).

$\mathrm{Hb}$, hemoglobin; LDH, lactate dehydrogenase; FLIPI, Follicular Lymphoma International Prognostic Index; Ki-67 PI, Ki-67 proliferation index; DLBCL, diffuse large B-cell lymphoma. 

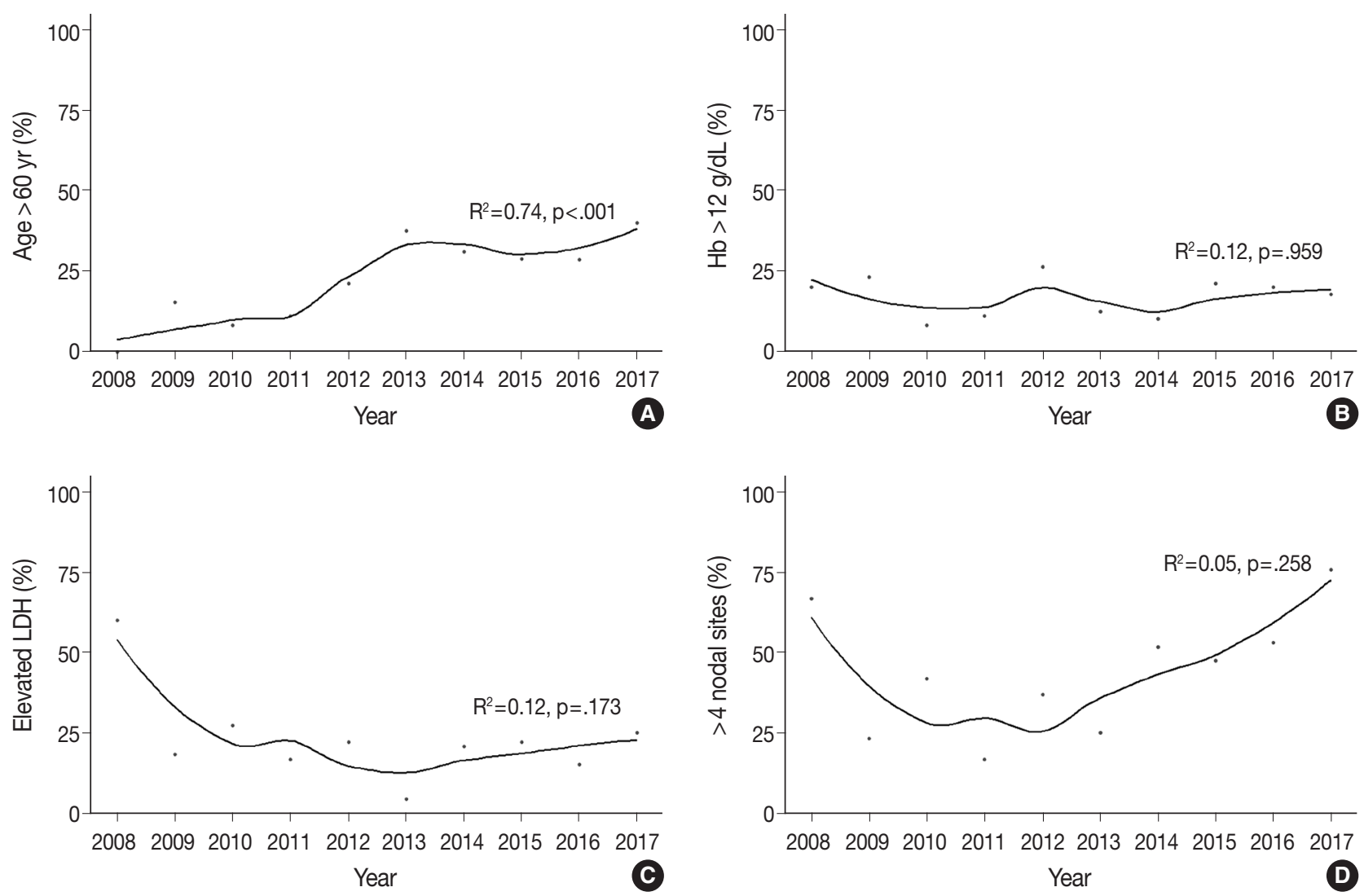

Fig. 2. Scatter plots with trend lines of the annual proportion of patients aged $>60$ years (A), with hemoglobin (Hb) level $<12 \mathrm{~g} / \mathrm{dL}$ (B), elevated lactate dehydrogenase (LDH) level (C), and involvement of more than four nodal sites (D).

years significantly increased between 2008 and $2017\left(R^{2}=0.74\right.$, $\mathrm{p}<.001)$ (Fig. 2A). In contrast, the rates of patients with $\mathrm{Hb}$ level $<12 \mathrm{~g} / \mathrm{dL}$ remained stable $\left(\mathrm{R}^{2}=-0.12, \mathrm{p}=.959\right)$ (Fig. 2B). The proportion of patients with elevated LDH level sharply decreased between 2008 and 2013 (60.0\% to 4.3\%) and gradually increased to $25.0 \%$ in 2017; however, these changes were not statistically significant $\left(\mathrm{R}^{2}=0.12, \mathrm{p}=.173\right)$ (Fig. $\left.2 \mathrm{C}\right)$. The proportion of patients with involvement of greater than four nodal sites rapidly decreased during 2008-2010 (66.7\% to $41.7 \%)$, but increased to $75.6 \%$ in $2017\left(\mathrm{R}^{2}=0.05, \mathrm{p}=.258\right)$ (Fig. 2D).

Over 10 years, the proportion of patients with stage III/IV disease significantly increased $\left(\mathrm{R}^{2}=0.36, \mathrm{p}=.039\right)$ (Fig. 3A), as was observed on the joinpoint regression analysis (AAPC, 5.0; 95\% CI, 0.4 to $9.7 ; \mathrm{p}=.035$ ) (Table 2). We found a decreasing trend in the number of cases of low-risk FLIPI $\left(\mathrm{R}^{2}=0.23, \mathrm{p}=\right.$ $.094)$ and a slightly increasing trend in the number of cases of grade 3B FL $\left(\mathrm{R}^{2}=-0.20, \mathrm{p}=.111\right)$, although the result was not statistically significant (Fig. 3B, C). Regarding histological pattern, the proportions of follicular pattern $\left(\mathrm{R}^{2}=0.24, \mathrm{p}=.086\right)$ and diffuse pattern $\left(\mathrm{R}^{2}=0.15, \mathrm{p}=.145\right)$ showed increasing trends, whereas follicular and diffuse patterns decreased $\left(R^{2}=0.34, p=\right.$ $.045)$ (Fig. 3D), and these variations were significant during 2008-2012 and 2013-2017 ( $\mathrm{p}=.018$ ) (Table 1).

For the IHC staining results, the rates of FL cases with BCL2 or BCL6 expression and those with $\mathrm{Ki}-67 \mathrm{PI} \geq 30 \%$ increased from 2008 to 2012 and from 2013 to 2017 ( $\mathrm{p}=.086, \mathrm{p}=.002$, and $\mathrm{p}=.016$, respectively) (Table 1 ). In the joinpoint regression analysis, the expression levels of BCL2 (AAPC, 2.6; 95\% CI, 0.7 to 4.6, $\mathrm{p}=.022$ ) and BCL6 (AAPC, 3.0; 95\% CI, 0.4 to 5.7; $\mathrm{p}=.028$ ) significantly increased over the 10 years (Table 2 ).

Since the 2000s, rituximab has been used widely, and a majority of the patients $(54.4 \%)$ in this study underwent rituximab-based chemotherapy; however, the proportion of patients on a watch-and-wait strategy significantly increased by $8.8 \%$ in 2008-2012 and by $22.2 \%$ in 2013-2017, while there was a slight decrease in the number of patients on rituximab-based therapy ( $\mathrm{p}=.049)$ (Table 1). There was no significant variation in other clinicopathologic features, e.g., sex, bone marrow involvement, transformation to DLBCL, and mortality, over the study period. 

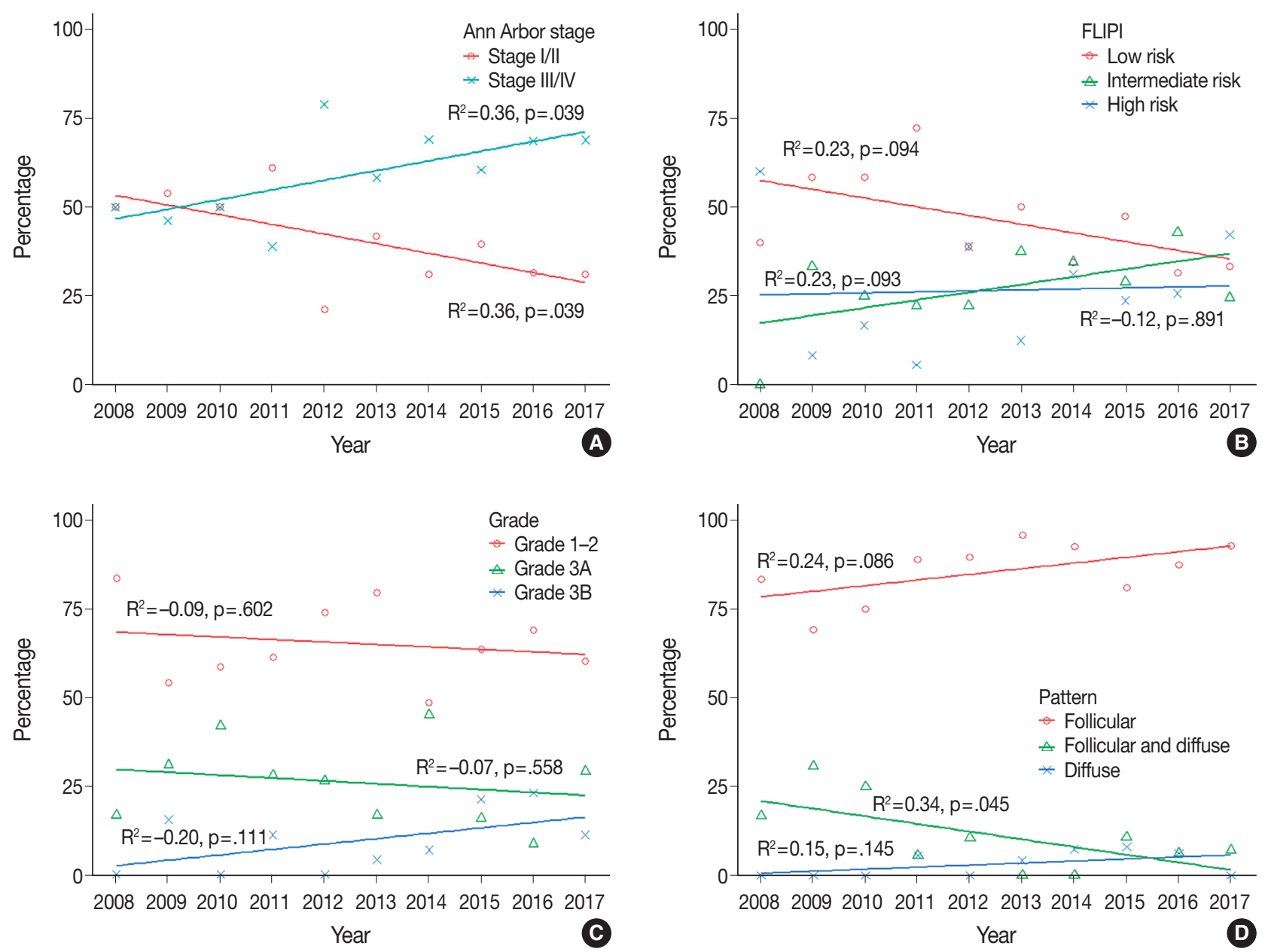

Fig. 3. Scatter plots with trend lines of the annual proportion of patients with follicular lymphoma: (A) Ann Arbor stage, (B) Follicular Lymphoma International Prognostic Index (FLIPI), (C) histologic grade, and (D) histologic pattern.

In survival analysis, during a median follow-up of 47 months (range, 0 to 222 months), 15 patients died, and the mean OS was 199 months. There was no significant OS difference between 2008-2012 and 2013-2017 (Supplementary Fig. S2).

\section{DISCUSSION}

The incidence rate of FL, which has been low in Korea, has increased in recent years. Lee et al. [9] studied 65,948 lymphoid diseases in the South Korean population and found increasing incidence of lymphoid malignancies $(6.85 \%$ to $13.18 \%)$ and FL $(0.22 \%$ to $0.39 \%)$ between 1999 and 2012. Kim et al. [27] studied epidemiologic changes in B-cell NHL in South Korea using the National Health Information Database and found that the incidence of FL has increased since 2006: the ratio of FL to NHL increased from 5.6\% to 7.8\% during 2006-2015, and the age-adjusted incidence of FL increased by $25 \%$ between
2011 and 2015. Similarly, in the current study, the incidence of FL significantly increased between 2008 and 2017, with an increasing ratio of FL:NHL. Although previous studies ascribed the low incidence of FL in Asian populations to ethnic differences, the recent trend of increased incidence rates might reflect the increasing adoption of a Westernized lifestyle by Asian populations $[6,28]$.

Currently, the etiology of FL is not understood fully; however, age, sex, ethnicity, genetic, clinical and environmental factors including lifestyle can affect the development of FL. Multiple large cohort studies have investigated the influence of smoking, alcohol intake, obese or overweight status and their relationship to FL development; however, the results have been conflicting [29]. A population-based study by Le et al. [30] in Canada during 1992-2010, demonstrated that the incidence rate of FL increased, especially in concentrated industrial zones, suggesting that industrial exposures play an important role in the develop- 
Table 2. Trends in clinicopathologic characteristics of FL: average annual percentage change

\begin{tabular}{|c|c|c|}
\hline Characteristic & AAPC $(95 \% \mathrm{Cl})$ & $p$-value \\
\hline Age $>60$ yr & $n a^{a}$ & \\
\hline $\mathrm{Hb}<12 \mathrm{~g} / \mathrm{dL}$ & $1.0(-0.91$ to 12.2$)$ & .833 \\
\hline Elevated LDH & $-6.5(-20.9$ to 10.5$)$ & .378 \\
\hline$>4$ nodal areas involved & 6.6 (-5.5 to 20.2) & .254 \\
\hline \multicolumn{3}{|l|}{ Ann Arbor stage } \\
\hline Stage $|/| \mid$ & $-6.2(-12.6$ to 0.7$)$ & .070 \\
\hline Stage III/IV & $5.0(0.4$ to 9.7$)$ & .035 \\
\hline Bone marrow involvement & $3.5(-5.9$ to 13.8$)$ & .455 \\
\hline \multicolumn{3}{|l|}{ FLIPI } \\
\hline Low & $-5.3(-10.8$ to 0.6$)$ & .071 \\
\hline Intermediate & $n a^{a}$ & \\
\hline High & $6.6(-12.4$ to 29.7$)$ & .486 \\
\hline \multicolumn{3}{|l|}{ Histologic grade } \\
\hline $1-2$ & $-0.9(-5.3$ to 3.7$)$ & .661 \\
\hline $3 \mathrm{~A}$ & $-4.7(-16.3$ to 8.6$)$ & .424 \\
\hline 3B & $n a^{a}$ & \\
\hline \multicolumn{3}{|l|}{ Histologic pattern } \\
\hline Follicular & $2.0(-0.3$ to 4.3$)$ & .120 \\
\hline Follicular and diffuse & $n a^{a}$ & \\
\hline Diffuse & $n a^{a}$ & \\
\hline BCL2-positive & $2.6(0.7$ to 4.6$)$ & .022 \\
\hline BCL6-positive & $3.0(0.4$ to 5.7$)$ & .028 \\
\hline CD10-positive & $1.0(-3.0$ to 5.2$)$ & .666 \\
\hline $\mathrm{Ki}-67 \mathrm{PI} \geq 30 \%$ & $5.4(-5.4$ to 17.3$)$ & .309 \\
\hline \multicolumn{3}{|l|}{ Treatment } \\
\hline Watch-and-wait & $n a^{a}$ & \\
\hline Rituximab-based & $2.1(-4.7$ to 9.4$)$ & .550 \\
\hline Radiation monotherapy & $n a^{a}$ & \\
\hline Transformation to DLBCL & $n a^{a}$ & \\
\hline
\end{tabular}

FL, follicular lymphoma; AAPC, average annual percent change; $\mathrm{Cl}$, confidence interval; $\mathrm{Hb}$, hemoglobin; LDH, lactate dehydrogenase; FLIPI, Follicular Lymphoma International Prognostic Index; Ki-67 PI, Ki-67 proliferation index; DLBCL, diffuse large B-cell lymphoma.

${ }^{\text {aNot }}$ applicable due to zero case counts for some years.

ment of FL. Further, it has been reported that exposure to herbicides is associated with FL by causing a t(14;18)-translocation [30].

In the current study, we found an increasing trend of stage III/IV disease over 10 years, and that the proportions of highrisk FLIPI and grade 3B FL also increased between 2008-2012 and 2013-2017, although the results were not statistically significant. In a study by Conconi et al. [14] that included $281 \mathrm{FL}$ cases from 1979 to 2007, the trend in the proportion of patients aged $\geq 60$ years with stage III/IV disease and high-risk FLIPI scores increased over the study period, although the result was not statistically significant. Mozas et al. [13] studied 727 grade 1-3A FL cases reported between 1980 and 2017, and found that grade 1-2 disease showed a decreasing trend over time, while high-risk FLIPI showed an increasing trend in recent years. These findings can be attributed to the increasing life expectancy and increase in the number of patients that present with advanced FL status at initial diagnosis, as a majority of the patients are asymptomatic.

We also note that most patients with FL have stage III/IV disease, a low-risk FLIPI score, and grade 1-2 FL, and the findings are comparable to those in the Unites States, showing predominance of stage III/IV, low-risk FLIPI score, and grade 1-2, with similar results to our data [29,31]. Cho et al. [5] retrospectively analyzed clinical characteristics and pathologic features of BCL2 expression in 343 FL patients in Korea during1993-2013, and demonstrated that predominancy of stage III/IV, low-risk FLIPI score, and grade 1-2 FL, suggesting that there was no significant difference between Korean and Western populations.

In this study, BCL2 or BCL6 immunopositivity significantly increased over the study period. We investigated whether the expression of BCL2 or BCL6 was associated with histologic grade; however, those unexpected findings were not associated with changes in the proportion of histologic grade (Supplementary Table S1). Although several studies have shown that the expression of $\mathrm{BCL} 2$ was less common in high-grade FL, the relationship between expression of BCL2 or BCL6 and histologic grade was not definitive in most prior studies [32-35].

The proportion of FL cases with Ki-67 PI $\geq 30 \%$ and diffuse pattern significantly increased between 2008-2012 and 20132017, and was significantly correlated with histologic grade (Supplementary Table S1). Most prior studies have shown that higher Ki-PI or diffuse pattern in FL generally correlates with higher grade or poor prognosis, although several FL cases with low grade and high Ki-PI have been described $[2,22,23,36]$. In this study, Ki-PI was estimated manually and might have produced intra- and interobserver variability. However, recent studies using software-automated quantification of Ki-67 PI in FL have shown that high Ki-67 PI was associated with grade $3 \mathrm{FL}$ or poor prognosis [19,21].

There were some limitations to this study. The low incidence of $\mathrm{FL}$ in our institutional series might have led to overestimation of the proportion of clinicopathologic features and inadequate statistical significance in the changes over the study period. FL has an indolent prognosis with a favorable outcome, and the number of events (e.g., death) per group was insufficient in the current study, in which large numbers of individuals were censored in the survival analysis. Thus, multicenter studies should be performed to precisely address the trends of FL in Korea. Our study was not a population-based study and lacks representativeness. However, we found an increase in the age-adjusted incidence rate of FL using data from a large tertiary institution, 
which is an accepted approach that can be adopted to allow better comparison [37,38]. In addition, the increasing incidence of $\mathrm{FL}$ and the ratio of $\mathrm{FL}$ to NHL observed in our data were similar to the trends observed in the National Health Information Database [27].

In conclusion, our study demonstrated an increase in the incidence rates of FL at a large tertiary institution in South Korea over the last 10 years, and we found recent changes in the clinicopathologic features of FL, an aspect that rarely has been studied.

\section{Supplementary Information}

The Data Supplement is available with this article at https://doi.org/10.4132/ jptm.2021.07.25.

\section{Ethics Statement}

All procedures performed in the current study were approved by the Institutional Review Board (IRB) of Asan Medical Center with a waiver of informed consent (IRB No. 2019-0509) and performed in accordance with the principles of the Declaration of Helsinki.

\section{Availability of Data and Material}

The datasets generated or analyzed during the study are available from the corresponding author on reasonable request.

\section{Code Availability}

Not applicable.

\section{ORCID}

Meejeong Kim https://orcid.org/0000-0002-1232-8439

Hee Sang Hwang https://orcid.org/0000-0001-9486-9214

Hyungwoo Cho https://orcid.org/0000-0003-4307-2795

Dok Hyun Yoon https://orcid.org/0000-0002-8289-3548

Cheolwon Suh https://orcid.org/0000-0002-9178-4431

Chan Sik Park https://orcid.org/0000-0001-9783-4498

Heounjeong Go https://orcid.org/0000-0003-0412-8709

Jooryung Huh https://orcid.org/0000-0002-2148-5778

\section{Author Contributions}

Conceptualization: MK, JH. Data curation: MK, HSH. Formal analysis: MK, HSH. Investigation: MK, HC, DHY, CS, HG. Methodology: MK, HSH, HC, HG. Project administration: HG, JH. Resources: MK, DHY, CS. Supervision: HG, CSP, JH. Validation: HSH, HG, CSP, JH. Visualization: MK, HSH. Writing_original draft: MK, HG, JH. Writing_review \& editing: MK, HSH, HG, CSP, JH. Approval of final manuscript: all authors.

\section{Conflicts of Interest}

H.G., a contributing editor of the Journal of Pathology and Translational Medicine, was not involved in the editorial evaluation or decision to publish this article. All remaining authors have declared no conflicts of interest.

\section{Funding Statement}

No funding to declare.

\section{References}

1. Freedman A. Follicular lymphoma: 2018 update on diagnosis and management. Am J Hematol 2018; 93: 296-305.

2. Swerdlow SH, Campo E, Harris NL, et al. WHO classification of tumours of haematopoietic and lymphoid tissues. Lyon: International Agency for Research on Cancer, 2017.

3. Guidelines for the diagnosis and treatment of follicular lymphoma in China. Cancer Biol Med 2013; 10: 36-42.

4. Yoshino T, Takata K, Tanaka T, Sato Y, Tari A, Okada H. Recent progress in follicular lymphoma in Japan and characteristics of the duodenal type. Pathol Int 2018; 68: 665-76.

5. Cho SH, Suh C, Do YR, et al. Clinical features and survival of patients with follicular lymphoma in Korea. Clin Lymphoma Myeloma Leuk 2016; 16: 197-202.

6. Intragumtornchai T, Bunworasate U, Wudhikarn K, et al. NonHodgkin lymphoma in South East Asia: an analysis of the histopathology, clinical features, and survival from Thailand. Hematol Oncol 2018; 36: 28-36.

7. Takata K, Miyata-Takata T, Sato Y, Yoshino T. Pathology of follicular lymphoma. J Clin Exp Hematop 2014; 54: 3-9.

8. Bai B, Huang HQ. Individualized management of follicular lymphoma. Chin Clin Oncol 2015; 4: 7.

9. Lee H, Park HJ, Park EH, et al. Nationwide statistical analysis of lymphoid malignancies in Korea. Cancer Res Treat 2018; 50: 222-38.

10. Yoo KH, Lee H, Suh C; CISL. Lymphoma epidemiology in Korea and the real clinical field including the Consortium for Improving Survival of Lymphoma (CISL) trial. Int J Hematol 2018; 107: 395-404.

11. Mustafa Ali M, Rybicki L, Nomani L, et al. Grade 3 follicular lymphoma: outcomes in the rituximab era. Clin Lymphoma Myeloma Leuk 2017; 17: 797-803.

12. Federico M, Caballero Barrigon MD, Marcheselli L, et al. Rituximab and the risk of transformation of follicular lymphoma: a retrospective pooled analysis. Lancet Haematol 2018; 5: e359-67.

13. Mozas P, Nadeu F, Rivas-Delgado A, et al. Patterns of change in treatment, response, and outcome in patients with follicular lymphoma over the last four decades: a single-center experience. Blood Cancer J 2020; 10: 31

14. Conconi A, Motta M, Bertoni F, et al. Patterns of survival of follicular lymphomas at a single institution through three decades. Leuk Lymphoma 2010; 51: 1028-34.

15. Karube K, Guo Y, Suzumiya J, et al. CD10-MUM1+ follicular lymphoma lacks BCL2 gene translocation and shows characteristic biologic and clinical features. Blood 2007; 109: 3076-9.

16. Hans CP, Weisenburger DD, Greiner TC, et al. Confirmation of the molecular classification of diffuse large B-cell lymphoma by immunohistochemistry using a tissue microarray. Blood 2004; 103: 27582 .

17. Shustik J, Han G, Farinha P, et al. Correlations between BCL6 rearrangement and outcome in patients with diffuse large B-cell lymphoma treated with CHOP or R-CHOP. Haematologica 2010; 95: 96101.

18. Miyaoka M, Kikuti YY, Carreras J, et al. Clinicopathological and genomic analysis of double-hit follicular lymphoma: comparison with high-grade B-cell lymphoma with MYC and BCL2 and/or BCL6 rearrangements. Mod Pathol 2018; 31: 313-26.

19. Kawaguchi Y, Shiozawa E, Shimada S, et al. Ki-67 expression of immunohistochemistry using computerized image analysis is a usefu prognostic marker in follicular lymphomas. Int J Clin Exp Pathol 2018; 11: 3366-74.

20. Szczuraszek K, Mazur G, Jelen M, Dziegiel P, Surowiak P, Zabel M. 
Prognostic significance of Ki-67 antigen expression in non-Hodgkin's lymphomas. Anticancer Res 2008; 28: 1113-8.

21. Samols MA, Smith NE, Gerber JM, et al. Software-automated counting of Ki-67 proliferation index correlates with pathologic grade and disease progression of follicular lymphomas. Am J Clin Pathol 2013; 140: 579-87.

22. Koster A, Tromp HA, Raemaekers JM, et al. The prognostic significance of the intra-follicular tumor cell proliferative rate in follicular lymphoma. Haematologica 2007; 92: 184-90.

23. Martin AR, Weisenburger DD, Chan WC, et al. Prognostic value of cellular proliferation and histologic grade in follicular lymphoma. Blood 1995; 85: 3671-8.

24. Kim HJ, Fay MP, Feuer EJ, Midthune DN. Permutation tests for joinpoint regression with applications to cancer rates. Stat Med 2000; 19: 335-51.

25. Picci P, Mercuri M, Ferrari S, et al. Survival in high-grade osteosarcoma: improvement over 21 years at a single institution. Ann Oncol 2010; $21: 1366-73$.

26. Shewale JB, Correa AM, Brown EL, et al. Time trends of perioperative outcomes in early stage non-small cell lung cancer resection patients. Ann Thorac Surg 2020; 109: 404-11.

27. Kim JS, Liu Y, Ha KH, Qiu H, Rothwell LA, Kim HC. Increasing incidence of B-cell non-Hodgkin lymphoma and occurrence of second primary malignancies in South Korea: 10-year follow-up using the Korean National Health Information database. Cancer Res Treat 2020; 52: 1262-72.

28. Biagi JJ, Seymour JF. Insights into the molecular pathogenesis of follicular lymphoma arising from analysis of geographic variation. Blood 2002; 99: 4265-75.

29. Ma S. Risk factors of follicular lymphoma. Expert Opin Med Diagn 2012; 6: 323-33.

30. Le M, Ghazawi FM, Alakel A, et al. Incidence and mortality trends and geographic patterns of follicular lymphoma in Canada. Curr Oncol 2019; 26: e473-81.

31. Friedberg JW, Taylor MD, Cerhan JR, et al. Follicular lymphoma in the United States: first report of the national LymphoCare study. J Clin Oncol 2009; 27: 1202-8.

32. Bilalovic N, Blystad AK, Golouh R, et al. Expression of bcl-6 and $\mathrm{CD} 10$ protein is associated with longer overall survival and time to treatment failure in follicular lymphoma. Am J Clin Pathol 2004; 121: 34-42.

33. Guo Y, Karube K, Kawano R, et al. Low-grade follicular lymphoma with $t(14 ; 18)$ presents a homogeneous disease entity otherwise the rest comprises minor groups of heterogeneous disease entities with $B c l 2$ amplification, $B c l 6$ translocation or other gene aberrances. Leukemia 2005; 19: 1058-63.

34. Schraders M, de Jong D, Kluin P, Groenen P, van Krieken H. Lack of $\mathrm{Bcl}-2$ expression in follicular lymphoma may be caused by mutations in the BCL2 gene or by absence of the $\mathrm{t}(14 ; 18)$ translocation. J Pathol 2005; 205: 329-35.

35. Choi SM, Betz BL, Perry AM. Follicular lymphoma diagnostic caveats and updates. Arch Pathol Lab Med 2018; 142: 1330-40.

36. Wang SA, Wang L, Hochberg EP, Muzikansky A, Harris NL, Hasserjian RP. Low histologic grade follicular lymphoma with high proliferation index: morphologic and clinical features. Am J Surg Pathol 2005; 29: 1490-6.

37. Yoon SO, Suh C, Lee DH, et al. Distribution of lymphoid neoplasms in the Republic of Korea: analysis of 5318 cases according to the World Health Organization classification. Am J Hematol 2010; 85: 760-4.

38. Sandvik OM, Soreide K, Kvaloy JT, Gudlaugsson E, Soreide JA. Epidemiology of gastrointestinal stromal tumours: single-institution experience and clinical presentation over three decades. Cancer Epidemiol 2011; 35: 515-20. 